\title{
AUTOMATIC GEOMETRICAL OPTIMIZATION BY WAY OF NUMERICAL FLOW MODELS
}

\author{
Erpicum S. ${ }^{1}$, Archambeau P. ${ }^{1}$, Dewals B. ${ }^{1,2}$, Pirotton M. ${ }^{1}$ \\ ${ }^{1}$ Hydrology, Applied Hydrodynamics and Hydraulic Constructions Research Unit (HACH), ArGEnCo Department, \\ University of Liege, Belgium, e-mail: s.erpicum@ulg.ac.be \\ ${ }^{2}$ National Fund for Scientific Research, FNRS-FRS, Belgium
}

\begin{abstract}
The potentialities in numerical flow modeling available today allow performing more and more representative and accurate computations of an increasing part of hydraulic engineering practical problems. In some cases, numerical simulation can be used as an alternative, or at least an efficient pre-design tool, to more classic physical modeling. In this framework, the paper presents the application of integrated numerical tools to the automatic optimization of the geometry of a guide-wall at the entrance of a channel. The simulations have been performed by using a Genetic Algorithm based optimization tool coupled to a 2D free surface flow model. This finite volume multibock flow solver, WOLF2D, solves the conservative form of the classical shallow water equations together with a depth-integrated $k-\varepsilon$ type turbulence model. The calculations have been performed on a cluster of 12 processors to reach a satisfactory solution in less than 2.5 days (96 solutions tested). On the basis of the numerical results and of the value of the suitability indicators, the optimal solution is objectively better than the other tested geometries. This confirms the efficiency of the automatic optimization procedure.
\end{abstract}

Key words Shallow water equations; Depth-averaged turbulence model; Automatic shape optimization; Genetic Algorithms

\section{INTRODUCTION}

In the framework of hydraulic structures design, the optimization of the geometry of the walls used to guide the water currents is still generally performed by the way of scale model studies. Indeed, the representativeness of the physical models, their reliability for all types of flows, providing that suited similarity laws are correctly applied, and their "as true as real" appearance make them the best tool to find the ideal shape and to persuade decision makers of the usefulness of most of the design adjustments.

Beside this classic and well known approach, the numerical flow modeling potentialities available today allow performing all or a part of this optimization work by way of an automatic procedure (Demny and al., 2003). Indeed, more and more representative and accurate flow solvers are available for more and more complex applications and the continuous increase in computers performance enable their use to solve an increasing part of the hydraulic engineering problems.

In this paper, the application of such numerical tools to the automatic optimization of the geometry of a guide-wall at the entrance of a channel is presented. The simulations are performed by using a Genetic Algorithm based optimization tool coupled to the free surface flow model WOLF2D.

The numerical tools are presented in a first time, before an explanation of the application characteristics and of the optimization procedure, 
performed on a cluster of 12 processors. The optimal solution is finally analyzed in comparison with other tested geometries.

\section{NUMERICAL MODELS}

\subsection{FLOW SOLVER}

The free surface flow model WOLF2D has been developed for more than 10 years at the University of Liege by the HACH team. It is based on the two-dimensional depth-averaged equations of volume and momentum conservation (SWE). In the "shallow-water" approach, the only assumption states that the velocity components normal to a main flow plane are significantly smaller than those in this plane. As a consequence, the pressure field is found to be almost hydrostatic everywhere.

The large majority of flows occurring in rivers, even highly transient, can be reasonably seen as shallow, except in the vicinity of some singularities (e.g. weirs). Indeed, vertical velocity components remain generally low compared to velocity components in the horizontal plane and, consequently, flows may be considered as mainly two-dimensional. Thus, the approach used in this paper is suitable for many of the problems encountered in river management for example.

In the numerical model, the bottom friction is conventionally modeled thanks to an empirical law, such as the Manning formula. The internal friction has to be reproduced by applying a proper turbulence model. A depth-integrated $k-\varepsilon$ type one, involving additional partial differential equations (Erpicum, 2006), has been used in this study.

The solver includes a mesh generator and deals with multiblock grids. Within each block, the grid is Cartesian to take advantage of the lower computation time and the gain in accuracy provided by this type of structured grids compared to unstructured ones (Erpicum and al., 2004). The multiblock feature increases the surface of domains discretizable with a constant cells number while enabling local mesh refinements close to interesting areas. It is thus a solution to solve the main drawback of Cartesian grid, i.e. the high number of cells they need to reach a fine enough discretization.

In addition, an automatic grid adaptation technique restricts the simulation domain to the wet cells to decrease the number of computation elements (Erpicum and al., 2004).

The space discretization is performed by means of a finite volume scheme. This ensures a proper mass and momentum conservation, which is a prerequisite for handling reliably discontinuous solutions such as moving hydraulic jumps. As a consequence, no assumption is required as regards the smoothness of the solution. Reconstruction at cells interfaces can be performed constantly or linearly, in conjunction with slope limiting, leading in the second case to a second-order spatial accuracy.

Since the model is applied to compute steadystate solutions, the time integration is performed by means of a 3-step first order accurate Runge-Kutta algorithm, providing adequate dissipation in time. For stability reasons, the time step is constrained by the Courant-Friedrichs-Levy condition based on gravity waves. A semi-implicit treatment of the bottom friction term is used, without requiring additional computational costs.

Slight changes in the Runge-Kutta algorithm coefficients allow modifying its dissipation properties and make it suitable for accurate transient computations.

\subsection{OPTIMIZATION TOOL}

The optimization module WOLF AG has been developed to provide a robust parameters fitting tool, usable with different flow solvers. It is based on the Genetic Algorithms method.

Genetic Algorithms are exploration algorithms imitating the natural selection and genetic 
mechanisms (Goldberg, 1989). At each step of the optimization process, they improve a set of several coded representations of the value of the parameters to be optimized, called a population of chromosomes or chains. Several genetic operators such as selection, crossover and mutation govern this evolution. These operators act in such a way that the best individuals of the existing populations are preferentially used to build the new ones. Thus, a kind of natural selection occurs.

The principle of evolution is quite simple (Erpicum and al., 2005). The process starts with a random initial population and the performance of all the different chains is evaluate thanks to a fitness function. Then, a new population is created chain by chain, using the following sequence. Two chains are selected in the initial population, with a probability directly proportional to their adaptation to the problem. The coding of these two chains (binary coding) is combined by exchanging parts of their elements (crossover). The only or multiple crossing places are chosen randomly, depending on the type of crossover and of the number of parameters. During the exchange of two elements, their value can mutate, with a very small probability.

This sequence is repeated until a new complete population is created. The whole creation process of a new population is called a generation. It is repeated until the optimum is reached.

Genetic Algorithms are obviously located between classic optimization methods, applied locally in the search space of solutions, and purely random or systematic optimization ones, that explore all the possible solutions. Genetic algorithms constitute a kind of intelligent and pseudo-random exploration of the space of solutions. Thus, they can be used to study discontinuous and disjointed functions and always converge towards the absolute optimum.
Genetics Algorithms can also be very easily parallelized. Indeed, to evaluate the efficiency of each generation, they require the computation of several problems differing only by the value of the parameters to be fitted. In this study, MPI libraries have been used to parallelize the solver on 12 processors (Erpicum, 2006).

\subsection{WOLF MODELING SYSTEM}

The numerical models used in this study are parts of the WOLF system, developed at the University of Liege for more than 10 years. WOLF includes a set of complementary and interconnected modules for simulating free surface flows: processoriented hydrology, 1D \& 2D hydrodynamic, sediment or pollutant transport, air entrainment.

Other functionalities of WOLF 2D include the use of moment of momentum equations (Dewals, 2006), the application of the cut-cell method (Erpicum and al., 2006) as well as computations considering vertical curvature effects by means of curvilinear coordinates in the vertical plane (Dewals and al., 2006).

A user-friendly GIS interface, entirely designed and implemented by the authors (Archambeau, 2006), makes the pre- and postprocessing operations very convenient. Import and export operations are easily feasible from and to various classical GIS tools. Different layers of maps can be handled to analyze information related to the topography, the ground characteristics, the vegetation density and the hydrodynamic fields.

\section{SHAPE OPTIMIZATION}

\subsection{DEFINITION OF THE PROBLEM}

The practical application presented below concerns the optimization of the shape of the left abutment of a channel junction in a reservoir. 
The channel starts from a reservoir to feed a hydropower plant located downstream. As the inclination of the channel direction along the reservoir bank is not suited, whirls appear in the channel, downstream of the confluence (Fig. 1). They decrease the active channel cross section, capture the filths floating at the reservoir surface and are thus undesirable.

A rehabilitation solution to avoid these whirls while keeping the channel orientation is to optimize the shape of the left abutment at the channel entrance, in order to guide the flow entering the channel along left bank. The design study has been carried out numerically and automatically with the solvers presented in this paper.

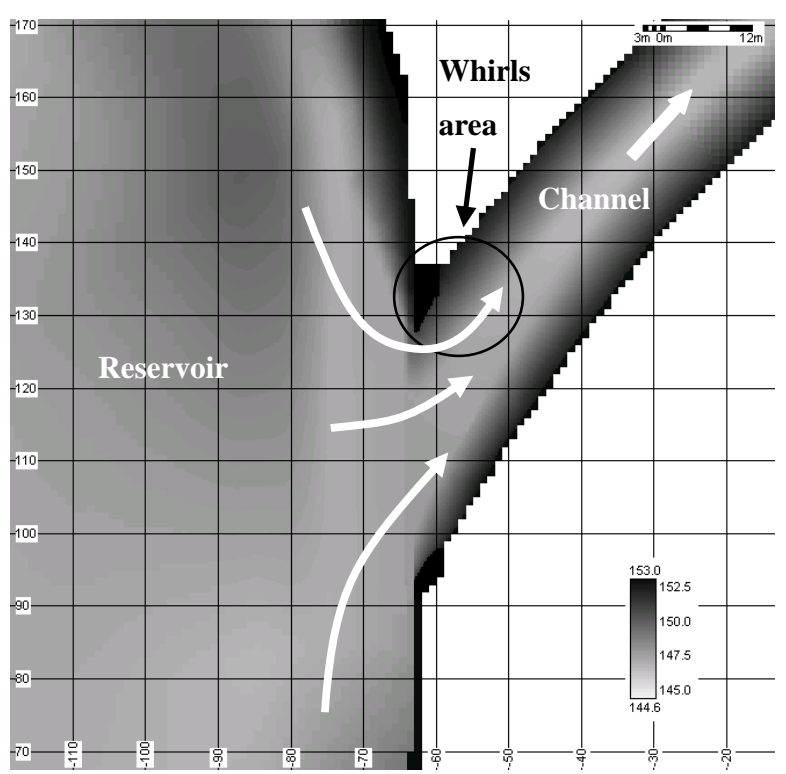

Fig. 1 Sketch of the reservoir and channel entrance Bathymetry in background (m)

\subsection{MODELING FEATURES}

\subsubsection{FLOW MODELING}

The numerical model covers a $1 \mathrm{~km}$ long reach of the reservoir and $250 \mathrm{~m}$ of the channel, with 142,000 square meshes from 1 to $.25 \mathrm{~m}$ shared out between 5 blocks. The finer grid has been used at the channel entrance while the coarser one has been applied upstream and downstream in the reservoir reach. The distributed bathymetry in the channel and the reservoir has been interpolated from cross section data, and building plans have been used to refine the junction topography. The roughness coefficient value has been chosen equal to $30 \mathrm{~m}^{1 / 3} / \mathrm{s}$.

The upstream boundary condition was the discharge in the reservoir $\left(240 \mathrm{~m}^{3} / \mathrm{s}\right)$. The downstream boundary condition was a water level in the reservoir reach (spillway) and the diverted discharge value at the channel downstream extremity $\left(70 \mathrm{~m}^{3} / \mathrm{s}\right)$.

All grid blocks have been used to compute the initial flow patterns and to define reliable hydrodynamics conditions at the boundary of the 3 blocks located around the channel entrance. Thus, the optimization simulations could focus on the most interesting area by computing the flow patterns only on the 75,000 meshes of the 3 blocks covering the channel and the reservoir right bank near the junction.

\subsubsection{OPTIMIZATION PARAMETERS}

3 parameters have been defined to optimize the shape of the left abutment at the channel entrance. They characterized 4 consecutive arcs of circle, creating a continuous line between the reservoir and the channel banks (Fig. 2). The 4 curves were tangent by pairs and the 2 extremity ones were tangent to the bank of the channel or reservoir. The 2 central curves had to include the extremity of the existing abutment (point 0 ).

The parameters were the $x$ and $y$ coordinates of the rotation center of arc 2 and the $y$ coordinate of the one of arc 3 . The 2 first parameters had to be in the rectangle $\mathrm{A}$ while the third one was limited to interval B (Fig. 2).

In the flow solver, during the optimization process, the topography of the area inside the curve defined by a set of parameters value was automatically modified to put it out of water and thus representing the specific abutment geometry. 


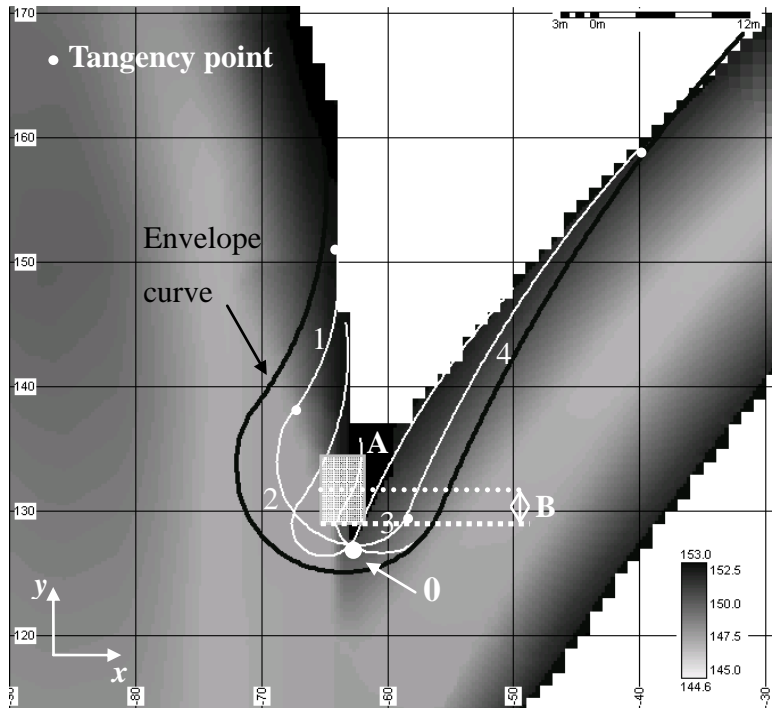

Fig. 2 Sketch of the left abutment shape and parameters interval for optimization

The most important problem of the application was the definition of the fitness function to be maximized by the best abutment geometry. The criterion was finally based on the uniformity of the flow velocity fields in 6 cross-sections in the channel (Fig. 3). For each section, after stabilization of the computed flow fields, the standard deviation of the perpendicular velocity distribution has been evaluated and the inverse of the higher value has been used as the fitness function value.

An efficient automatic criterion has to be defined to assess the stabilization of the hydrodynamic simulations. Indeed, these computations are rather time consuming and, in order to accelerate the optimization process, it is thus very important to stop them immediately when the stable solution is reached.

Every 15 seconds, the relative variation along time of the specific discharges was evaluated in 17 points spread around the abutment (Fig. 3). If the whole of these points had an evolution lower than $.5 \%$, the hydrodynamic simulation was stopped. An additional criterion consisting in a maximum time modeling has been added to prevent a non stabilization of a solution. In this last case, the fitness function value of the set of parameters was artificially decreased to be seen as a solution to be rejected.

Initial flow conditions have been defined in agreement with all the possible abutment geometries: the specific discharge fields have been defined for the maximum abutment surface while the water heights have been computed considering the smaller abutment geometry. Initial conditions were thus suited for all abutment geometries.

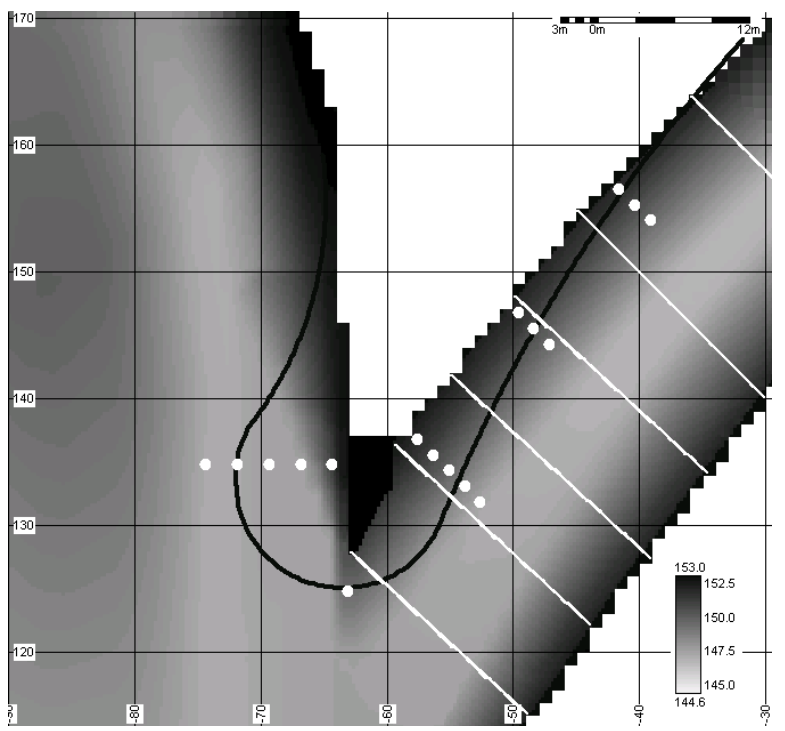

Fig. 3 Sections to evaluate the fitness function and points to assess computation transient state

\subsection{RESULTS}

Populations of 12 chains have been used to perform the optimization. The whole of the fitness functions of one population were evaluated in the order of 8 hours on a cluster of 12 processors. The parameter coding enabled to test 1024 different solutions.

After 8 generations (96 solutions tested), the optimum illustrated in Fig. 4 has been reached. It provides the most uniform velocity fields across the 6 sections defined in the channel, and it is thus objectively better than all the 95 other geometries tested by the solver. The flow lines represented on Fig. 4 show that the optimal geometry avoids the formation of whirls. 

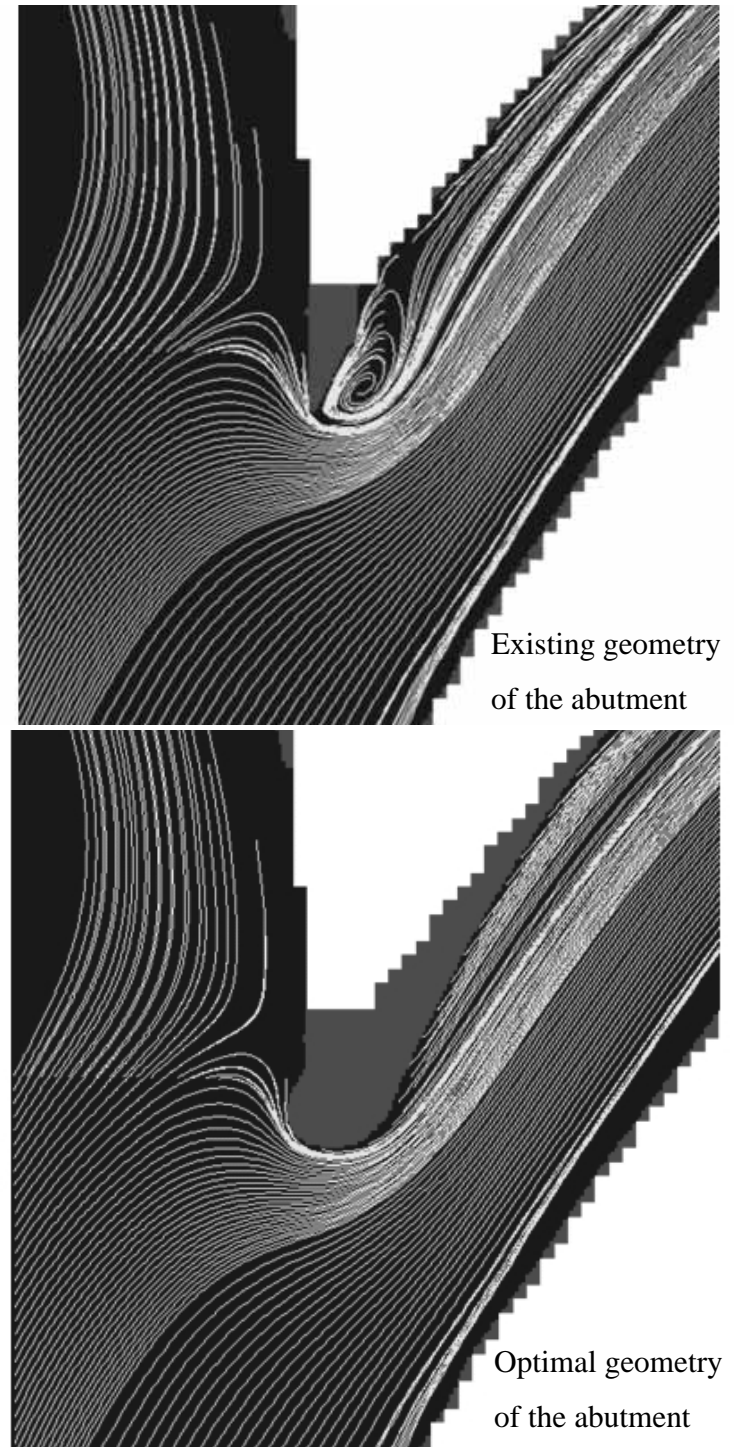

Fig. 4 Flow lines for the existing (above) and optimal (below) left abutment geometry

\section{CONCLUSION}

By using a 2D free surface flow solver linked to a Genetic Algorithms based optimization tool, the geometry of a guide-wall located at the entrance of a channel has been automatically optimized by way of numerical modeling in less than 3 days on a cluster of 12 processors.

The final result fulfills all of the optimization criteria and is objectively better than the other tested solutions.

\section{Acknowledgements}

The authors gratefully acknowledge the Administration des Ponts et Chaussées, Luxembourg, and the Bureau Lombardi SA Ingénieurs Conseils, Switzerland, for supplying hydraulic and topography data used in this study.

\section{REFERENCES}

Archambeau P. (2006) Contribution à la modélisation de la genèse et de la propagation des crues et inondations. $\mathrm{HACH}, \mathrm{PhD}$ Thesis, Université de Liège, Liège.

Demny, G., C. Homann, C. Shoemaker and J. Köngeter. (2003) Automatic optimization of hydraulic structures. in Proc. $30^{\text {th }}$ IAHR Congress. Thessaloniki, Greece

Dewals B.J. (2006) Une approche unifiée pour la modélisation des écoulements à surface libre, de leur effet érosif sur une structure et de leur interaction avec divers constituants. $\mathrm{HACH}, \mathrm{PhD}$ Thesis, Université de Liège, Liège.

Dewals B.J., Erpicum S., Archambeau P., Detrembleur S., and Pirotton M. (2006) Depth-integrated flow modeling taking into account bottom curvature. Journal of Hydraulic Research, Vol. 44(6). 787-795.

Erpicum S., Archambeau P., Dewals B.J., Detrembleur S., Fraikin C. and Pirotton M. (2004) Computation of the Malpasset dam break with a 2D conservative flow solver on a multiblock structured grid. In Proc. of $6^{\text {th }}$ Int. Conf. on Hydroinformatics, Liong, Phoon and Babovic (eds), World Scientific Publishing Company

Erpicum S., Archambeau P., Dewals B., Detrembleur S. and Pirotton M. (2005) Optimization of hydroelectric power stations operations with WOLF package, in Hydropower'05 - The backbone of sustainable energy supply. Stavanger, Norway.

Erpicum S. (2006) Optimisation objective de paramètres en écoulements turbulents à surface libre sur maillage multibloc. HACH, PhD Thesis, Université de Liège, Liège.

Erpicum S., Archambeau P., Dewals B.J., Detrembleur S., and Pirotton M. (2006) Fluid-structure interaction modeling with a coupled 1D-2D finite volume free surface flow solver. In RiverFlow 2006, Ferreira,Alves, Leal and Cardoso (eds), Taylor \& Francis: London.

Goldberg D.E. (1989) Genetic Algorithms in search, optimization and machine learning. Addison-Wesley. 